This Author Accepted Manuscript (AAM) is published in:

Nature Energy, vol. 1, 16134 (2016)

DOI: $10.1038 /$ nenergy.2016.134

\title{
A regenerative elastocaloric heat pump
}

\author{
Jaka Tušek $^{\mathrm{a}, \mathrm{b}}$, Kurt Engelbrecht ${ }^{\mathrm{a}}$, Dan Eriksen ${ }^{\mathrm{a}}$, Stefano Dall’Olio ${ }^{\mathrm{a}}$, Janez Tušek ${ }^{\mathrm{b}}$ and Nini \\ Pryds*,a
}
a Technical University of Denmark, Department of Energy Conversion and Storage, Riso Campus, Frederiksborgvej 399, DK-4000, Roskilde, Denmark
${ }^{\mathrm{b}}$ University of Ljubljana, Faculty of Mechanical Engineering, Aškerčeva 6, SI-1000
Ljubljana, Slovenia
* Corresponding author
e-mail: jaka.tusek@fs.uni-lj.si; kuen@dtu.dk; nipr@dtu.dk

A large fraction of global energy use is for refrigeration and air-conditioning, which could be decarbonised if efficient renewable energy technologies could be found. Vapour-compression technology remains the most widely used system to move heat up the temperature scale after more than 100 years; however, caloric-based technologies (those using the magnetocaloric, electrocaloric, barocaloric or elastocaloric effect) have recently shown a significant potential as alternatives to replace this technology due to high efficiency and the use of green solid-state refrigerants. Here, we report a regenerative elastocaloric heat pump that exhibits a temperature span of $15.3 \mathrm{~K}$ on the water side with a corresponding specific heating power up to $800 \mathrm{~W} \mathrm{~kg}$ and maximum COP (coefficient-of-performance) values of up to 7. The efficiency and specific heating power of this device exceeds those of other devices based on caloric effects. These results open up the possibility of using the elastocaloric effect in various cooling and heatpumping applications.

Demand for heating and cooling is rapidly increasing and accounts for a significant portion of the total world energy demand. After over 100 years as the most commonly used cooling technology, vapour-compression devices remain the most popular method to move heat through a closed-looped cycle. These devices are characterised by relatively low efficiency and have detrimental effects on the environment when their refrigerant is released into the atmosphere. Therefore, a new technology is needed. Over the past decade, a large scientific effort has been applied to the development of alternative cooling and heat-pumping technologies based on so-called caloric effects (magnetocaloric, electrocaloric, barocaloric and elastocaloric effect) in ferroic materials ${ }^{1-5}$. It was shown that these solid-state technologies can potentially be more efficient and more environmentally friendly than vapour-compression ${ }^{6}$. Among these technologies, the one based on the elastocaloric effect (eCE) shows the highest theoretical potential performance due to the large available latent heat $^{7,8}$. However, these advantages have not yet been proven experimentally, although a substantial worldwide effort has been recently exerted towards the development of elastocaloric devices ${ }^{9-11}$.

The eCE is closely related with the martensitic transformation of shape memory alloys (SMA), in particular with their superelastic effect. The superelastic effect is associated with the reversible, stress-induced austenitic-martensitic transformation, which is the basis for the 
This Author Accepted Manuscript (AAM) is published in:

Nature Energy, vol. 1, 16134 (2016)

DOI: $10.1038 /$ nenergy.2016.134

elastocaloric thermodynamic cycle. When an SMA in the austenite phase is strained/stressed, an exothermic process occurs. The transformation releases heat or, under adiabatic conditions, heats the material. When the stress is removed, the reverse transformation occurs, which is endothermic (under adiabatic conditions the material cools down), and the material transforms back to the austenite phase. The reverse transformation is usually associated with a stress hysteresis in the transformation region. Such a cooling/heat-pumping thermodynamic cycle is shown schematically in Supplementary Fig. 1 and explained in detail in refs. 8, 12. In general, all SMAs can be considered as potential elastocaloric materials if their transformation temperatures (i.e. austenitic finish temperature) are below the operating temperature of the device, which is a precondition for reversible superelasticity. The most widely used SMA is binary $\mathrm{Ni}-\mathrm{Ti}$, which was first reported in $1963^{13}$ and remains by far the most widely applied SMA due to its superior fatigue behaviour and its biocompatibility ${ }^{14}$. Recently, Ni-Ti has attracted significant attention for its possible application in efficient and environmentally friendly cooling or heat pump applications due to its high adiabatic temperature changes over a wide temperature range near room temperature ${ }^{12,15-17}$. In recent years, a significant research effort has focused on the development of new Ni-Ti-based alloys, such as $\mathrm{Ni}-\mathrm{Ti}-\mathrm{Cu}-\mathrm{Co}(\mathrm{Fe})$ and $\mathrm{Ni}-\mathrm{Ti}-\mathrm{Cu}-\mathrm{V}$ alloys, which have shown improved structural and functional stability and reduced hysteresis, while still exhibiting a significant $\mathrm{eCE}^{18,19}$. In particular, Ni-Ti-Cu-Co alloys show no fatigue and good functional stability even after 10 million loading-unloading cycles, which already fulfil the demand for commercial elastocaloric applications ${ }^{18}$. Other SMAs, including $\mathrm{Cu}$-based (e.g., $\mathrm{Cu}-\mathrm{Zn}-\mathrm{Al}^{20}$ ), Fe-based (e.g., Fe-Pd ${ }^{21}$ ), magnetic SMAs ${ }^{22,23}$ and shape memory polymers ${ }^{24}$ also show a significant eCE. A comprehensive review of elastocaloric materials and systems can be found in refs. $25,26$.

Concurrently with the research and development on elastocaloric materials, significant progress was made in design and development of elastocaloric devices in the last year ${ }^{26}$. In 2015, two single-stage cooling/heat-pumping systems based on a single elastocaloric plate loaded in tension with mechanical travel between heat sink and heat source utilising contact heat transfer were presented ${ }^{9,10}$. The maximum temperature span measured was approximately $7 \mathrm{~K}$ for both devices. Such a concept was suggested to be applied for novel MEMS (microelectromechanical system) applications, such as temperature control of lab-on-chip systems or micro cooling in bio-medical applications ${ }^{10}$. Furthermore, a cooling system applying $\mathrm{Ni}$ - $\mathrm{Ti}$ tubes loaded in compression and arranged into double porous structures through which heat transfer fluid is pumped using a heat recovery principle was presented in ref. 11. The initial experimental results show a temperature span of $1.5 \mathrm{~K}$ on the fluid side ${ }^{11}$, however it was shown numerically that further improvements on the systems are possible ${ }^{27}$. In spite of the developments reported so far, a new development concept that can bring elastocaloric cooling closer to different commercial applications is needed.

In this work we present a concept for an elastocaloric device using the principle of active regeneration. An analogue system, called the active magnetic regenerator, has been extensively used for more than two decades to utilise the magnetocaloric effect in magnetic refrigeration ${ }^{6}$. However, this principle has not yet been demonstrated for elastocaloric systems. Interestingly, our elastocaloric regenerator constructed of a $\mathrm{Ni}$ - $\mathrm{Ti}$ alloy produced exceptionally high performance with a temperature span of $15.3 \mathrm{~K}$ on the water side, a corresponding specific heating power reaching a value of $800 \mathrm{~W} \mathrm{~kg}^{-1}$ and a maximum COP (coefficient-of-performance) of 7 (depending on operating conditions). These results have already surpassed most of the existing devices based on caloric effects (in particular magnetic 
This Author Accepted Manuscript (AAM) is published in:

Nature Energy, vol. 1, 16134 (2016)

DOI: $10.1038 /$ nenergy.2016.134

refrigeration, which is the most mature technology) and demonstrate a great potential for this technology to be applied in cooling and heat pump applications in the near future.

\section{Operating principle of a regenerative elastocaloric device}

A schematic of the regenerative elastocaloric device and its hydraulic system are shown in Fig. 1. The central component of the system is a regenerator made of elastocaloric elements (plates in this case) arranged in a porous structure. The regenerator is at both ends clamped in mechanical grips which are attached to the actuator or similar system that loads and unloads the regenerator. The regenerator is connected on both sides to two fluid loops (namely, the hot and cold fluid loops) with hot and cold external heat exchangers (HHEX and CHEX), which reject and absorb heat from the surroundings, respectively. The system includes a small actuator attached to a displacer (piston) to circulate the fluid, four check valves, which control the fluid flow direction, and four thermocouples to monitor the system temperature.

The principle of operation is as follows (in the case of a Brayton-like thermodynamic cycle ${ }^{26}$ ). The first step in the process is loading (straining the material) of the regenerator in the absence of fluid flow, which causes the material to heat up due to the eCE. In the second step, a piston moves the fluid through check valve V1, through the regenerator that is at an elevated temperature (where the fluid heats up) and further through check valve V4 towards the HHEX, where heat is rejected to the surroundings. In the third step, the regenerator is unloaded (released), which causes the material to cool down, due to the eCE, and in the final step, the piston pumps the fluid in the counter flow direction (through check valves V3 and V2) towards CHEX, where the cooled fluid can now absorb heat from the load. These four steps are repeated continuously, establishing a temperature profile along the length of the regenerator (in the fluid flow direction). This thermodynamic cycle enables a temperature span between the hot and cold side that can be several times the adiabatic temperature change of the elastocaloric material. This method of utilising elastocaloric materials opens up the possibility to apply elastocaloric technology in practical applications where a certain cooling/heating load at an operating temperature span of $15 \mathrm{~K}$ or more is generally required.

\section{Construction of a regenerator}

The elastocaloric regenerator has to meet several important requirements in order to work efficiently as an active regenerator system. The first precondition is a sufficient fatigue life. If this technology is to reach the market, the required fatigue life for a commercial application is likely to be above 10 million cycles (i.e. several years of operation), which can be reached with advanced elastocaloric materials such as $\mathrm{Ni}-\mathrm{Ti}-\mathrm{Co}-\mathrm{Cu}$ alloys ${ }^{18}$. The second precondition is to assure a homogeneous stress and strain distribution throughout the regenerator, i.e. a uniform strain of each plate. A non-uniform strain distribution among the plates could result in local heat sinks and heat sources that can disrupt the temperature gradient in the flow direction. On the other hand, it is known that the strain distribution and related adiabatic temperature change in the material can be highly nonhomogeneous, with Lüders-like bands ${ }^{28}$ in particular at applied strains that do not result in a complete transformation. This can also reduce the performance of the elastocaloric regenerator and must be addressed in the future with different stabilization techniques capable of homogenizing the eCE throughout the material ${ }^{12,19,29}$. The third precondition is that the materials in the regenerator should be sufficiently thin with small spacing between them to assure fast and efficient heat transfer from the material to the heat transfer fluid. This was shown to be of an extreme importance to 
ensure efficient operation of the active magnetic regenerator ${ }^{30}$. In order to fulfil these requirements a regenerator with thin dog-bone shaped $\mathrm{Ni}$ - $\mathrm{Ti}$ plates loaded in tension was constructed by stacking the plates and laser welding them, which was shown to provide an equal strain distribution and equal eCE among all the plates in the regenerator (see Supplementary Fig. 6). In order to increase fatigue life, the maximum applied strain in the regenerator was limited to $4 \%$, which does not correspond to a fully complete transformation as shown in Supplementary Figure 5. Prior to implementation of the elastocaloric regenerator in the device, it was mechanically trained to stabilize its superelastic behaviour ${ }^{12,31}$ in order to assure reproducible operation of the device (see Supplementary Fig. 5). After the training period, the regenerator was sealed with a flexible housing composed of flax fibres impregnated with silicone and sealed with a final silicone layer. The housing materials accommodate the relatively large regenerator deformation in the loading direction while withstanding the forces exerted by the flowing heat transfer fluid and additionally providing some thermal insulation. On each regenerator end, two fluid ports were attached in order to connect the regenerator to the fluid system and to ensure that the flow in each tube in the flow system is unidirectional. The details of the regenerator fabrication, stabilization and evaluation of its eCE can be found in the Methods section and Supplementary Information. A comprehensive analysis of the Ni-Ti plates used in the device can be found in ref. 32, while the adiabatic temperature changes of the plate as a function of applied strain are shown in Supplementary Fig. 7 (redrawn from ref. 32). The elastocaloric regenerator applied in the device is shown in Fig. 2a. Figure $2 b$ shows the envelope of the temperature span between the hot and cold side of the regenerator until steady state conditions are reached (measured with the thermocouples), while Figs. 2c-f shows an example of the operation of the device captured with an IR camera from the initial state until steady state conditions are reached, where a temperature profile established along the length of the regenerator is visible. A photo of the entire elastocaloric device together with the hydraulic set-up is shown in Supplementary Fig. 4.

\section{Heat pump characteristics}

For the purposes of demonstrating the system, we operated the system under heat pump conditions. The choice of operating mode depends strongly on the type of materials used. In the current demonstration, the austenitic finish temperature of the Ni-Ti alloy was $280 \pm 5 \mathrm{~K}$ (based on the material supplier specifications), which also determined the lower operating temperature of the device. At any stage in the cycle the regenerator temperature should not be below the material's austenitic finish temperature, which will cause bending of the Ni-Ti plates as described in detail in ref. 8 due to the incomplete austenite-martensite transformation. The overall heat pump performance characteristics of the elastocaloric device are shown in Fig. 3. The system was evaluated at two operating frequencies and four applied strains (at a constant volume of fluid pumped through the regenerator in each cycle). The details of the operating conditions for testing and a detailed description of the calculations, i.e. the temperature span, specific heating power and the COP values, are given in the Methods section. It should be noted that COP values reported here do not include inefficiencies in the mechanical actuator and force recovery system or pumping power, therefore, it is to be expected that actual COP values will be somewhat lower. As seen in Fig. 3, higher frequencies generate higher temperature spans and higher heating powers because the elastocaloric material is cycled more frequently, meaning there is more eCE per time. On the other hand, the higher frequency generally results in lower COP values, as has also been observed for magnetic refrigeration ${ }^{6}$. The temperature span and the heating power increase 
with the applied strain as expected due to the higher eCE, while the COP values decrease with strain since the input work increases more rapidly compared to the heating power. The largest temperature span between the hot and cold side was measured to be $15.3 \mathrm{~K}$ with a corresponding specific heating power of $782 \mathrm{~W} \mathrm{~kg}^{-1}$ of the active elastocaloric material and a COP value of 3.5 (at an operating frequency of $0.25 \mathrm{~Hz}$ and applied strain of $3.4 \%$ ). At a smaller strain $(1.7 \%)$ a COP of 5.5 was measured at a temperature span of $6.5 \mathrm{~K}$ and a corresponding specific heating power of $355 \mathrm{~W} \mathrm{~kg}^{-1}$. Even higher COP values (up to 7) were obtained at higher flow rates, as shown in the Supplementary Fig. 10. It should be noted that here the input mechanical work was assumed to be equal to the enclosed area in the stressstrain diagram (see Supplementary Fig. 8). It is assumed that the work released during unloading can be fully recovered, which can be realized by simultaneous loading of another regenerator operating in tandem as shown for example in the single-stage elastocaloric device ${ }^{9}$.

\section{Discussion}

Comparing these results with numerical modelling results of the regenerative elastocaloric cycle $^{8}$, it can be seen that even higher temperature spans and larger thermal loads can be achieved. Therefore, we believe that there is still plenty of room for improvements e.g. the system performance, in particular with higher operating frequency and better regenerator geometry (thinner plates and smaller hydraulic diameter) ${ }^{32}$, but also with better thermal isolation and a smaller thermal mass of the hydraulic system. Nonetheless, our technological advancement of the field is only the beginning and paves the way for development of newer and better devices in the future. A special focus in the future should be placed on design and development of the most suitable driving system that provides large forces and small displacements at high efficiency. This will be one of the crucial elements in efficient and commercially relevant elastocaloric devices. A review and comparison of possible driving systems applicable to elastocaloric device are presented ref. 26.

Finally, the combination of an effective regenerative device and elastocaloric materials with high adiabatic temperature change enables the realization of a highly efficient cooling/heating device using a regenerative cycle. Implementations that realize such a regenerative cycle which enables temperature spans of the device that significantly exceed the temperature spans measured on any other developed elastocaloric systems (see Fig. 4a) and can open up the opportunity to implement this technology in a future commercial device. Its heat pump performance also exceeds the cooling performance (on a mass of solid refrigerant basis) of a great majority of magnetic (magnetocaloric) refrigerators published to date (see Fig. 4b). As a first report of an elastocaloric regenerative device, we could only compare its heat pumping performance with magnetic refrigeration, which is the most mature and most studied in the past two decades among all caloric technologies.

In conclusion, it was shown that the elastocaloric effect can produce outstanding results when applied in a regenerative cycle. The most promising result in this study is the excellent heat pumping performance at a significant temperature span. In addition, a great advantage of the elastocaloric technology is its significantly lower price of materials compared to most of other caloric materials (especially magnetocaloric materials). The results obtained for this elastocaloric system are extremely promising, showing a great potential for this technology to be applied in various renewable energy applications in our daily-based life, such as household appliances, industrial cooling, transport cooling, automotive air-conditioning, etc. 
This Author Accepted Manuscript (AAM) is published in:

Nature Energy, vol. 1, 16134 (2016)

DOI: $10.1038 /$ nenergy.2016.134

\section{Methods}

Elastocaloric regenerator fabrication. Polycrystalline $\mathrm{Ni}$-Ti sheets with a thickness of $0.2 \mathrm{~mm}$, mass fraction of $\mathrm{Ni}_{0.558} \mathrm{Ti}_{0.442}$ and austenitic finish temperature of $280 \pm 5 \mathrm{~K}$ were obtained from Memry $\mathrm{GmbH}$. An electron backscatter diffraction analysis of these materials revealed a well-developed $\{111\}$ (uvw) texture, which is frequently observed in rolled $\mathrm{Ni}_{-} \mathrm{Ti}^{29}$. The plates were further cut into dog-bone shaped samples according to ASTM standard E8/E8M (see Supplementary Fig. 2 for sample dimensions) using the electrical discharge machining (EDM) technique such that the strain is applied in the rolling direction, which results in a larger transformation plateau than the normal to rolling direction ${ }^{41}$. To remove imperfections and cracks from the edges of the plate which lead to premature fatigue failure, the plates were mechanically polished prior to construction of the regenerator. The mechanical polishing consisted of starting from fine grit sandpaper and moving to wax compounds and a buffing wheel. Polishing was performed in the direction of strain application to avoid minor cracks in the direction perpendicular to the strain direction. Nine polished plates were then assembled into a stack together with spacers to create fluid flow channels (see Supplementary Fig. 3). The spacing between the plates was held constant at $0.2 \mathrm{~mm}$ which resulted in a regenerator porosity of $50 \%$. The total mass of the active material in the regenerator was $5.8 \mathrm{~g}$. In order to assure an equal stress distribution among all the plates in the stack, the plates and the spacing were welded together into a rigid structure at the gripping area using laserwelding technology (see Supplementary Fig. 3). Geometrical properties of the elastocaloric regenerator system are presented in Supplementary Table 1. Welding has been performed with a pulsed laser Nd: YAG with an average power of $420 \mathrm{~W}$ and no filler material. Initially, the spot welding was performed in nine equally distributed spots over each gripping area of the stack as shown in Supplementary Fig. 3. Approximately half of the plates in the stack (dog-bone shaped plates and spacers) were welded (in a single shot) from one side, while the rest of the plates were welded from the other side. The highest power of the laser pulse was limited in order to prevent sputtering of the molten weld pool. At the beginning, the pulse (peak) power was $5.5 \mathrm{~kW}$, followed by an exponential decrease with a pulse time of $18 \mathrm{~ms}$. The exponential decrease of the pulse power was required to assure simultaneous cooling of the welds and its surroundings in order to increase its toughness. The pulse frequency was $2 \mathrm{~Hz}$ with a total welding time at one spot of $1 \mathrm{~s}$. The diameter of the laser beam in focus was 1.5 $\mathrm{mm}$ with a Gaussian energy distribution. The applied focal length was $160 \mathrm{~mm}$. In addition to spot welding, the stack was further welded at the edges of the gripping area with short continuous welds. In this case, the pulse frequency was $5 \mathrm{~Hz}$ with a peak power of $2.1 \mathrm{~kW}$ (followed by an exponential decrease) and pulse duration of 10 ms. In order to seal the regenerator and while maintaining enough flexibility to be compatible with the superelastic regenerator, the regenerator was sealed with flexible housing materials made of natural fibre impregnated with silicone that is wrapped orthogonally to the direction of strain. The fluid ports attached to each regenerator end were made using the Polyjet 3D printing process. Thermocouples were mounted in the fluid flow at each fluid inlet/outlet in the system. They were mounted as close to the regenerator as possible to reduce effects of heat losses from the pipes to ambient.

Stabilization of the regenerator. The mechanical training (stabilization) of the regenerator as well as further experimental characterization of the elastocaloric device were performed on a Zwick/Roell EZ030 electromechanical testing actuator equipped with mechanical T-fatigue grips. It has an Xforce K load cell that has a 30 $\mathrm{kN}$ capacity with an uncertainty of $1 \%$. The strain was measured based on the displacement of the crosshead, which has an accuracy of $\pm 1 \mu \mathrm{m}$, thereby measuring global or average strain over the sample. Since the sample width and therefore the strain was not constant over the length of the sample, the strains reported here should be considered estimated values based on the equivalent gauge length $(70 \mathrm{~mm})$. The training of the regenerator was performed at room temperature $\left(22^{\circ} \mathrm{C}\right)$ with 200 quasi-isothermal loading-unloading cycles at a strain rate of $0.000143 \mathrm{~s}^{-1}$ up to a strain of $4 \%$ (see Supplementary Fig. 5).

Elastocaloric device characterization. The elastocaloric device was tested under different operation conditions. We evaluated two operating frequencies $(0.125 \mathrm{~Hz}$ and $0.25 \mathrm{~Hz})$ and for each frequency, four different strains were tested $(1.7 \%, 2.3 \%, 2.8 \%$, and 3.4\%) - see Supplementary Fig. 8. For example, in the case of $0.25 \mathrm{~Hz}$, each step in the process (loading-fluid flow-unloading-fluid flow) takes one second, while in the case of 0.125 $\mathrm{Hz}$, each step takes two seconds. The applied strain rate was therefore not constant and can be calculated for each test based on the (un)loading duration and the applied strain. The volume of the fluid pumped through the regenerator was held constant at the volume of the fluid inside the regenerator $\left(800 \mathrm{~mm}^{3}\right)$, so the entire fluid volume inside the regenerator was exchanged during a single fluid flow period. This was decided based on pretests which showed that this is approximately the optimal value with respect to the temperature span (see Supplementary Fig. 10). The fluid (water) was pumped through the regenerator with piston displacers 
This Author Accepted Manuscript (AAM) is published in:

Nature Energy, vol. 1, 16134 (2016)

DOI: $10.1038 /$ nenergy.2016.134

incorporated in the hydraulic system that were moved with an actuator. The mass flow rate was calculated based on the piston movement per fluid flow period. The device performance was evaluated based on the temperature measurements from the thermocouples (type $\mathrm{K}$ with a wire diameter of $0.25 \mathrm{~mm}$ using a National Instruments 9213 thermocouple module) placed at all four fluid ports at the inlet/outlet of the regenerator. The sampling frequency of the data was $10 \mathrm{~Hz}$. The temperature measurements and the timing control of both actuators were performed with LabView software and a National Instruments data acquisition system. The thermocouples were calibrated in situ and the device operates in a temperature-controlled room and the uncertainty of the thermocouples is estimated as $0.3 \mathrm{~K}$. For visualization purposes (since the emissivity of the device was not controlled) the operation of the device was captured with an IR camera (FLIR SC5200). The regenerator underwent more than 2,000 loading-unloading cycles (up to different strains) and did not fail during the experimental characterization.

Calculation of the temperature span, specific heating power and COP values. The system performance was evaluated when steady-state conditions were reached (temperature of the fluid at the exit and entrance of the regenerator did not change with time). The temperature span of the device is defined as the temperature difference between the fluid's temperature at the exits of the hot and cold sides (measured with thermocouples) see Supplementary Fig. 9a. The specific heating power $\left(q_{h}\right)$ and the COP are defined by the following equations:

$$
\begin{gathered}
q_{h}=\frac{1}{m} \cdot \frac{P_{f}}{P_{\text {tot }}} \cdot \dot{m}_{f} \cdot c_{p, f} \cdot\left(T_{\text {hot }, \text { out }}-T_{\text {hot }, \text { in }}\right)=\frac{1}{m} \cdot \frac{P_{f}}{P_{\text {tot }}} \cdot \dot{m}_{f} \cdot c_{p, f} \cdot \Delta T_{h o t} \\
C O P=\frac{q_{h}}{w_{\text {mech }}}
\end{gathered}
$$

Where $m$ is the mass of active elastocaloric materials in the system (in this case $5.8 \mathrm{~g}$ ); $P_{f}$ is the duration of the fluid flow period; $P_{t o t}$ is the duration of the total cycle; $\dot{m}_{f}$ is the mass flow rate of the fluid flow calculated based on the piston movement per fluid flow duration; $c_{p, f}$ is the specific heat of the fluid (water); $\Delta T_{h o t}$ is the temperature drop in the hot fluid loop (temperature difference between the fluid at the exit and entrance of the hot side of the regenerator) noted in Supplementary Fig. 9a; $w_{\text {mech }}$ is the specific mechanical input work calculated based on the enclosed area in the stress-strain diagram shown in Supplementary Fig. 8 and calculated as:

$$
w_{\text {mech }}=\frac{1}{\mathrm{~m}} \cdot v \cdot V \oint \sigma \cdot d \varepsilon
$$

Where $v$ is the frequency of operation (number of cycles per unit of time); $V$ is the volume of the elastocaloric material in the structure; $\sigma$ is stress and $\varepsilon$ is strain. It should be noted that the input work required to pump the fluid through the regenerator can be neglected when compared to the mechanical work and was therefore not included in the COP calculation.

\section{References}

1. Fähler S. et al. Caloric effects in ferroic materials: New concepts for cooling. $A d v$. Eng. Mater. 14, 10-19 (2012).

2. Mañosa, L., Planes, A. \& Acet, M. Advanced materials for solid-state refrigeration. $J$. Mater. Chem. A 1, 4925-4936 (2103).

3. Moya, X., Kar-Narayan, S. \& Mathur, N.D. Caloric materials near ferroic phase transitions. Nature Mater. 13, 439-450 (2014).

4. Moya, X., Defay, E., Heine, V. \& Mathur, N.D. Too cool to work. Nature Phys. 11, 202-205 (2015).

5. Takeuchi I. \& Sandeman K. Solid-state cooling with caloric materials. Physics Today 68, 48-54 (2015).

6. Kitanovski A. et al. Magnetocaloric energy conversion: From theory to applications (Springer International Publishing, Switzerland, 2015)

7. Goetzler, W., Zogg, R., Young, J. \& Johnson, C. Energy Savings Potential and R\&D Opportunities for Non-Vapor-Compression HVAC Technologies (Navigant Consulting, Inc., prepared for U.S. Department of Energy, 2014) 
8. Tušek, J. et al. The Elastocaloric effect: A way to cool efficiently. Adv. Ener. Mater. 5, 1500361 (2015).

9. Schmidt, M., Schütze, A. \& Seelecke, S. Scientific test setup for investigation of shape memory alloy based elastocaloric cooling processes. Int. J. Refrig. 54, 88-97 (2015).

10. Ossmer, H., Miyazaki, S. \& Kohl, M. Elastocaloric heat pumping using a shape memory alloy foil device. In 2015 Transducers - 2015 18th International Conference on Solid-State Sensors, Actuators and Microsystems, 726-729 (Anchorage, USA, 2015).

11. Qian, S. et al. Design, development and testing of a compressive thermoelastic cooling system. In International Congress of Refrigeration 2015 (Yokohama, Japan, 2015).

12. Tušek, J., Engelbrecht, K., Mikkelsen, L.P. \& Pryds N. Elastocaloric effect of Ni-Ti wire for application in a cooling device. J. Appl. Phys.,117, 124901 (2015).

13. Buehler, W. J., Gilfrich, J. W. \& Wiley, R. C. Effects of low-temperature phase changes on the mechanical properties of alloys near composition TiNi. J. Appl. Phys., 34, 1475-1477 (1963).

14. Jani, J. M., Leary, M., Subic, A. \& Gibson, M. A. A review of shape memory alloy research, applications and opportunities. Mater. Design 56, 1078-1113 (2014).

15. Cui, J. et al. Demonstration of high efficiency elastocaloric cooling with large $\Delta \mathrm{T}$ using NiTi wires. Appl. Phys. Lett. 101, 073904 (2012).

16. Ossmer, H. et al. Evolution of temperature profiles in TiNi films for elastocaloric cooling. Acta Mater. 81, 9-20 (2014).

17. Pataky, G.J., Ertekin, E. \& Sehitoglu. H. Elastocaloric cooling potential of NiTi, Ni2FeGa, and CoNiA. Acta Mater. 96, 420-427 (2015).

18. Chluba, C. et al. Ultralow-fatigue shape memory alloy films. Science 348, 1004-1007 (2015).

19. Schmidt, M. et al. Thermal stabilization of NiTiCuV shape memory alloys: observations during elastocaloric training. Shap. Mem. Superelasticity 1, 132-141 (2015).

20. Mañosa, L., Jarque-Farnos, S., Vives, E. \& Planes, A. Large temperature span and giant refrigerant capacity in elastocaloric $\mathrm{Cu}-\mathrm{Zn}-\mathrm{Al}$ shape memory alloys. Appl. Phys. Lett. 103, 211904 (2013).

21. Xiao, F., Fukuda, T., Kakeshita T. \& Jin X. Elastocaloric effect by a weak first-order transformation associated with lattice softening in an Fe-31.2Pd (at.\%) alloy. Acta Mater. 87, 8-14 (2015).

22. Millán-Solsona, R. et al. Large entropy change associated with the elastocaloric effect in polycrystalline Ni-Mn-Sb-Co magnetic shape memory alloys. Appl. Phys. Lett. 105, 241901 (2014).

23. Sun, W., Liu, J., Lu, B., Li, Y. \& Yan, A. Large elastocaloric effect at small transformation strain in $\mathrm{Ni}_{45} \mathrm{Mn}_{44} \mathrm{Sn}_{11}$ metamagnetic shape memory alloys. Scripta Mater. 114, 1 (2016).

24. Xie, Z., Sebald, G. \& Guyomar, D. Elastocaloric effect dependence on pre-elongation in natural rubber. Appl. Phys. Lett. 107, 081905 (2015).

25. Lu, B. \& Liu J. Mechanocaloric materials for solid-state cooling. Sci. Bull. 60, 16381643 (2015).

26. Qian, S. et al. A review of elastocaloric cooling: materials, cycles and system integrations. Int. J. Refrig. 64, 1-19 (2016).

27. Qian, S. et al. Performance enhancement of a compressive thermoelastic cooling system using multi-objective optimization and novel designs. Int. J. Refrig. 57, 62-76 (2015). 
28. Sittner, P., Liu, Y. \& Novak, V. On the origin of Lüders-like deformation of NiTi shape memory alloys. J. Mech. Phys. Solids 53, 1719-1746 (2005).

29. Engelbrecht, K. et al. Effects of surface finish and mechanical training on Ni-Ti sheets for elastocaloric cooling. APL Mater. 4, 064110 (2016).

30. Tušek, J., Kitanovski, A. \& Poredoš A. Geometrical optimization of packed-bed and parallel-plate active magnetic regenerators. Int. J. Refrig. 36, 1456-1464 (2013)

31. Miyazaki, S., Imai, T., Igo, Y., \& Otsuka, K. Effect of Cyclic Deformation on the Pseudoelasticity Characteristics of Ti-Ni Alloys. Metall. Trans. A 17, 115-120 (1986).

32. Tušek, J., Engelbrecht, K. \& Pryds, N. Elastocaloric effect of a Ni-Ti plate to be applied in a regenerator-based cooling device. Accepted for publication in Sci. Technol. Built. Environ. (2016).

33. Zimm, C. et al. Description and performance of a near-room temperature magnetic refrigerator. Adv. Cryog. Eng. 43, 1759-1766 (1998).

34. Hirano, N. et al. Development of magnetic refrigerator for room temperature application. In Advances in Cryogenic Engineering: Proceedings of the Cryogenic Engineering Conference, Vol 47, 1027-1034 (2002).

35. Tura, A. \& Rowe, A. Permanent magnet magnetic refrigerator design and experimental characterization. Int. J. Refrig. 34, 628-639 (2011).

36. Jacobs, S. et al. The performance of a large-scale rotary magnetic refrigerator. Int. J. Refrig. 37, 84-91 (2014).

37. Bahl, C.R.H. et al. Development and experimental results from a $1 \mathrm{~kW}$ prototype AMR. Int. J. Refrig. 37, 78-83 (2014).

38. Eriksen, D. et al. Design and experimental tests of a rotary active magnetic regenerator prototype. Int. J. Refrig. 58, 14-21 (2015).

39. Arnold, D.S., Tura, A., Ruebsaat-Trott, A. \& Rowe A. Design improvements of a permanent magnet active magnetic refrigerator. Int. J. Refrig. 37, 99-105 (2014).

40. Aprea, C., Greco, A., Maiorino, A. \& Masselli, C. The energy performances of a rotary permanent magnet magnetic refrigerator. Int. J. Refrig. 61, 1-11 (2016).

41. Liu, Y. The superelastic anisotropy in a NiTi shape memory alloy thin sheet. Acta Mater. 95, 411-427 (2015).

\section{Acknowledgements}

Jaka Tušek would like to acknowledge the support of DTU's international H.C. Ørsted postdoc program and the Slovenian Research Agency (project no. Z2-7219) for supporting this work. The authors would like to thank TKC d.o.o., Ljubljana for performing the laserwelding of the elastocaloric regenerators.

\section{Author contributions}

J.T, K.E and N.P conceived the idea, designed the experiment and wrote the paper. D.E and S.D helped with the experimental design and running the experiments. J.T. managed the laser welding of the elastocaloric regenerator. N.P supervised the project.

\section{Additional information}

Supplementary information is linked to the online version of the paper at www. nature.com/nature. Correspondence and requests for materials should be addressed to J.T, K.E and N.P. 
This Author Accepted Manuscript (AAM) is published in:

Nature Energy, vol. 1, 16134 (2016)

DOI: $10.1038 /$ nenergy.2016.134

\section{Competing interests}

The authors declare no competing financial interests.

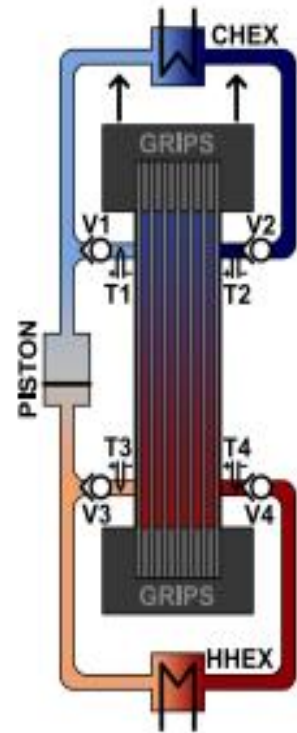

a

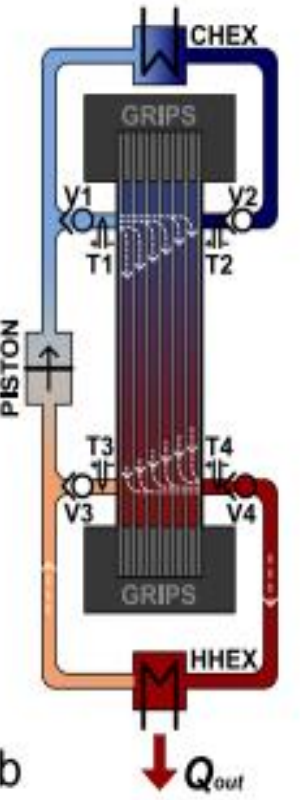

$\supset$ thermocouple (T)

C

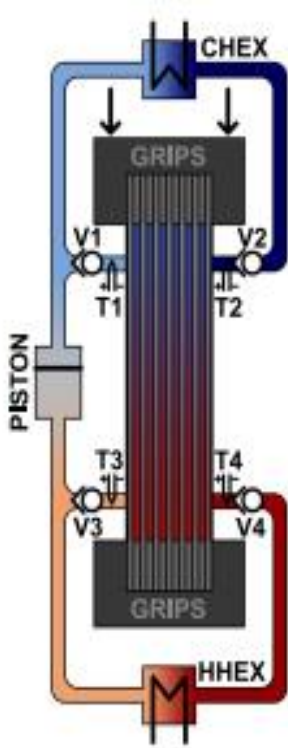

check valve (V) b

$$
\text { - }
$$

Figure 1. Illustration of four operational steps of the elastocaloric regenerator-based device. a, Loading - the length of the regenerator is increased causing the martensitic transformation. b, Fluid flow towards HHEX - where heat is rejected to the surroundings. c, Unloading - the regenerator is returned to the initial length causing the reverse martensitic transformation. d, Fluid flow towards CHEX - where heat is absorbed from the load. Symbols

$\mathrm{V} 1, \mathrm{~V} 2, \mathrm{~V} 3$ and V3 presents four check valves, while T1, T2, T3 and T4 presents four thermocouples placed at all four inlets and outlets of regenerator to controls fluid flow direction and measure temperature, respectively. Colour coding represents the established temperature profile along the system - at the hot side (noted with red) heat is rejected to the surroundings $\left(Q_{o u t}\right)$ through the hot heat exchanger (HHEX), while at the cold side (noted with blue) heat is absorbed from the load $\left(Q_{i n}\right)$ through the cold heat exchanger (CHEX). At both ends the regenerator is clamped in mechanical grips. 

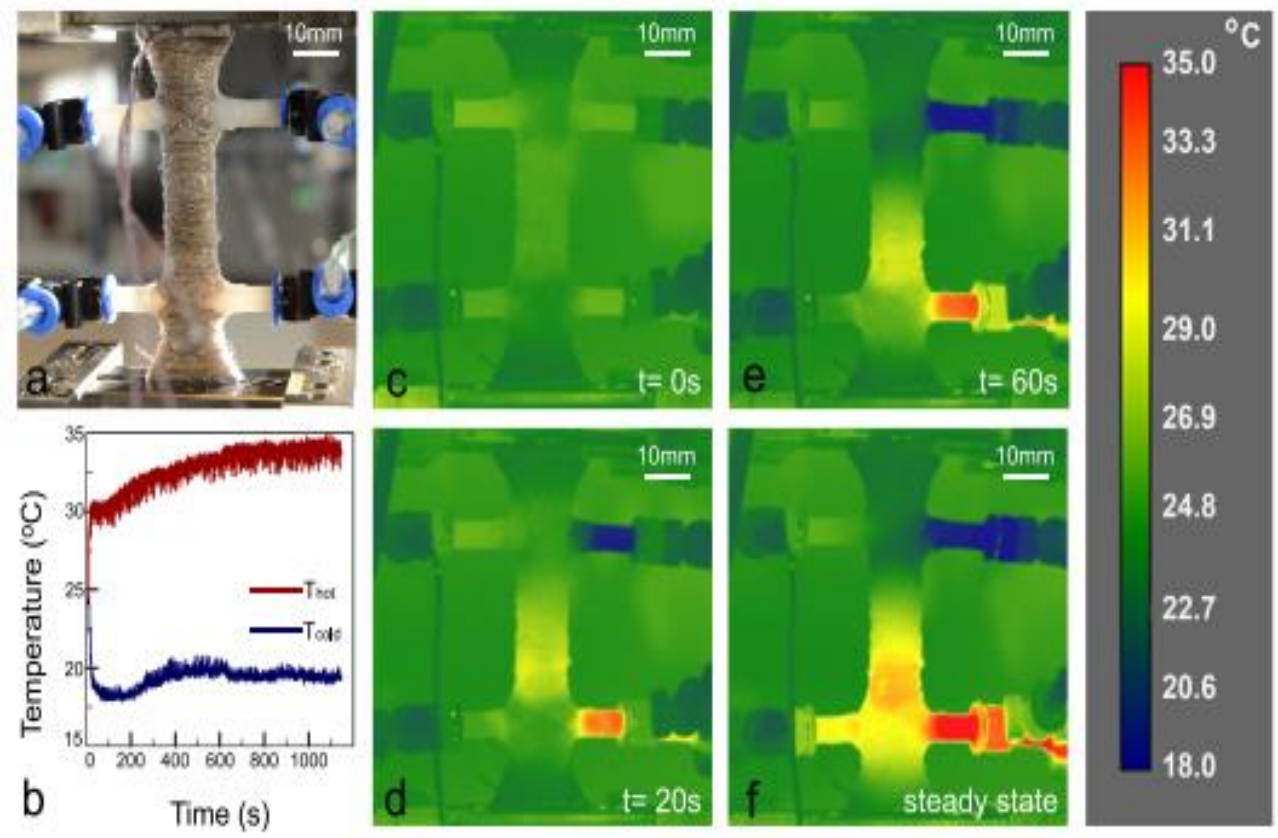

Figure 2. Elastocaloric regenerator and an example of its operation. a, A photo of the regenerator clamped in the mechanical grips and connected to the fluid loops (with noted fluid-flow directions). $\mathbf{b}$, The envelope of the temperature span between the hot and cold side of the regenerator until steady state conditions are reached. $\mathbf{c}-\mathbf{f}$, The regenerator in operation captured with an IR camera at four different sequences from the initial state till the steady state conditions are reached (for video of the operation see Supplementary Video 1).

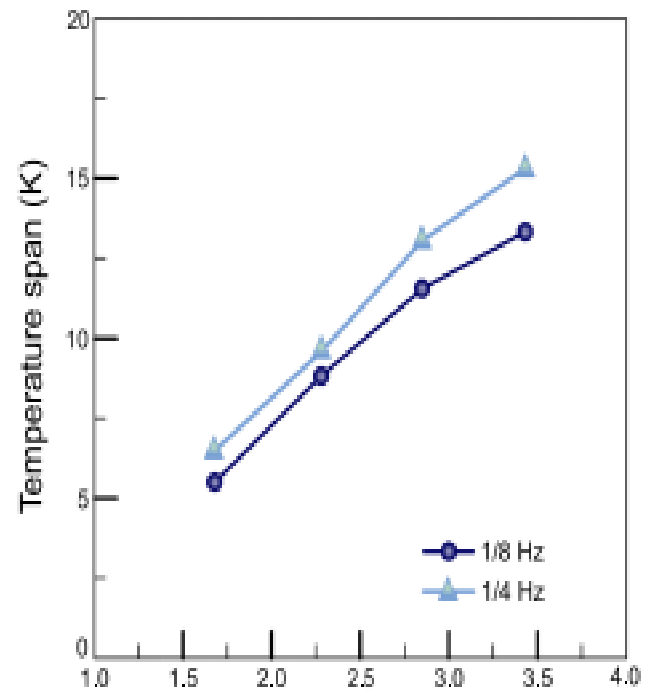

a Strain $(\%)$

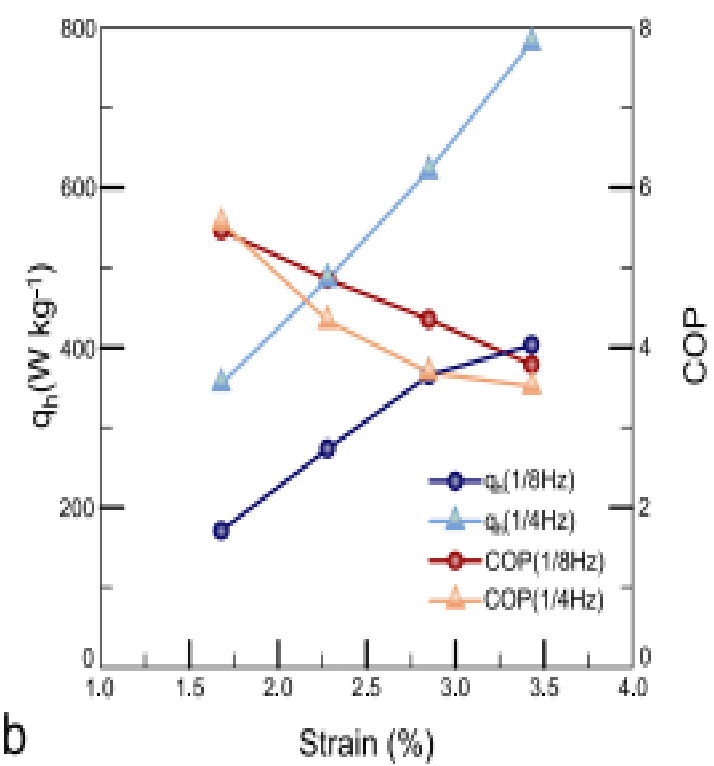

Strain (\%)

Figure 3. Performance of the elastocaloric device at different operation conditions. a, The maximum temperature span of the system as a function of the applied strain at two operating frequencies. $\mathbf{b}$, The corresponding specific heating power $\left(q_{h}\right)$ and COP values. 


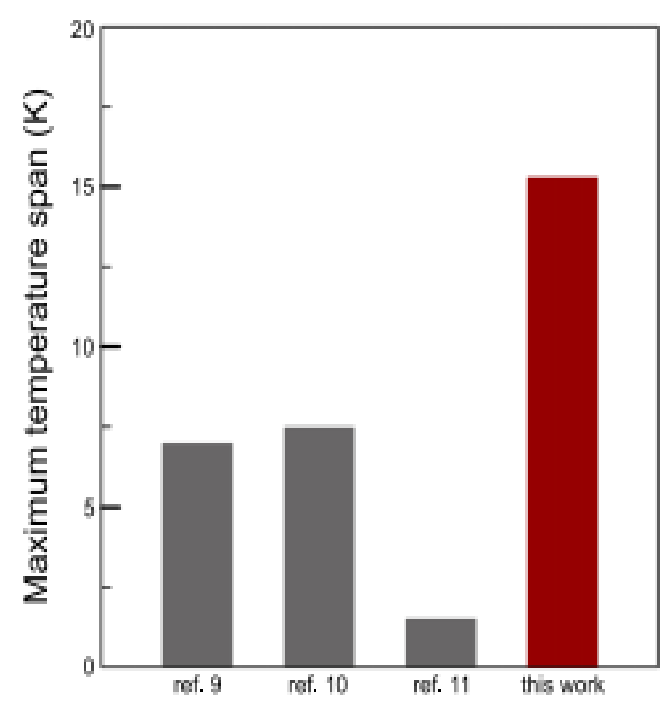

a

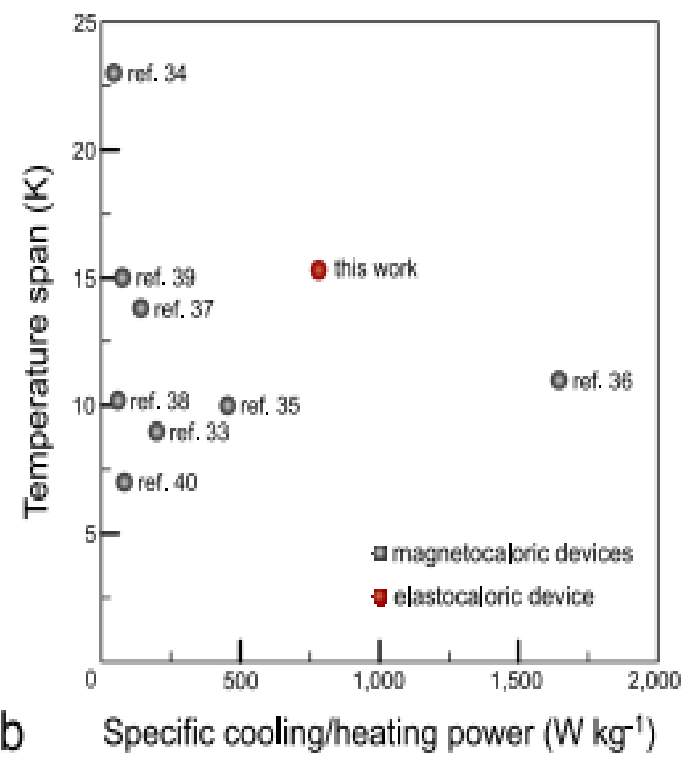

Figure 4. Comparison of the performance of the developed elastocaloric device with other reported caloric-based systems. a, A comparison of the maximum temperature span measured on the developed elastocaloric device with other elastocaloric systems from literature. $\mathbf{b}$, A performance comparison of the developed elastocaloric device with the eight best magnetic refrigerators (magnetocaloric devices) reported operating at their optimal conditions for practical applications that require high temperature span and high corresponding specific cooling/heating power. 